Submitted to Phys. Rev. A. $3^{\text {rd }}$ July, 1996

\title{
Separation of the Exchange-Correlation Potential into Exchange plus Correlation: an Optimized Effective Potential Approach
}

\author{
Claudia Filippi \\ Laboratory of Atomic and Solid State Physics and Cornell Theory Center, Cornell University, Ithaca, New York 14853 \\ C. J. Umrigar \\ Cornell Theory Center and Laboratory of Atomic and Solid State Physics, Cornell University, Ithaca, New York 14853 \\ Xavier Gonze \\ Unité P.C.P.M., Université Catholique de Louvain, B-1348 Louvain-la-Neuve, Belgium
}

\begin{abstract}
Most approximate exchange-correlation functionals used within density functional theory are constructed as the sum of two distinct contributions for exchange and correlation. Separating the exchange component from the entire functional is useful since, for exchange, exact relations exist under uniform density scaling and spin scaling. In the past, accurate exchange-correlation potentials have been generated from essentially exact densities but they have not been correctly decomposed into their separate exchange and correlation components (except for two-electron systems). Using a recently proposed method, equivalent to the solution of an optimized effective potential problem with the corresponding orbitals replaced by the exact Kohn-Sham orbitals, we obtain the separation according to the density functional theory definition. We compare the results for the $\mathrm{Ne}$ and $\mathrm{Be}$ atoms with those obtained by the previously used approximate separation scheme.
\end{abstract}

Keywords: density-functional theory, exchange-correlation potential

\section{INTRODUCTION}

Within density functional theory (DFT), the ground state energy of an interacting system of electrons in an external potential can be written as a functional of the ground state electronic density [1]. In the Kohn-Sham formulation of density functional theory [2], the ground state density is obtained as the density of a system of non-interacting electrons in an effective local potential. Although density functional theory is in principle exact, the energy functional contains an unknown quantity, called the exchange-correlation energy, $E_{\mathrm{xc}}[\rho]$. The effective potential for the fictitious noninteracting system is the sum of the external potential, the Hartree potential and the exchange-correlation potential, which is the functional derivative with respect to the density of $E_{\mathrm{xc}}[\rho]$. The density functional theory definition of the separate exchange and correlation components of $E_{\mathrm{xc}}[\rho]$ is based on the non-interacting system and is such that the resulting exchange functional has properties that are useful guides in the construction of an approximate exchange. Consequently, most approximate exchange-correlation functionals are also constructed as the sum of two distinct contributions for exchange and correlation.

In the past, exchange-correlation potentials and energies, of varying degrees of accuracy, have been determined by generating a density for the system of interest and then computing an exchange-correlation potential that yields the desired density as the ground state solution for the fictitious non-interacting system. In this context, researchers have used charge densities calculated by quantum chemistry methods for atoms [3 13] and molecules [14 16], as well as Quantum Monte Carlo methods for atoms [17,19] and for a model semiconductor [18]. The subsequent inverse problem, namely the search of the corresponding exchange-correlation potential, has been performed using a variety of different techniques. For example, the exchange-correlation potential has been determined by expanding it in a set of basis functions and varying the expansion coefficients to reproduce the accurate density [5, 19]. 
With the exception of two-electron systems, these accurate exchange-correlation potentials have never been separated into their exchange and correlation components according to the density functional theory definition. For manyelectron systems, an approximate scheme was used where the exchange potential was defined as the potential yielding the Hartree-Fock density and the correlation potential as the difference of the accurate exchange-correlation potential and this approximate exchange potential. In this paper, following the approach proposed by Görling and Levy [22], we obtain for the first time the correct separation of accurate exchange-correlation potentials for the Be atom and the Ne atom.

In Sec. II, we briefly introduce density functional theory and its Kohn-Sham formulation. In Sec. III, we derive the formulae used to determine the decomposition of the exchange-correlation potential into exchange and correlation. A comparison with approximate separation schemes is given in Sec. IV. In Appendix A, we describe the method for the special case of closed shell systems.

\section{THEORETICAL BACKGROUND}

Density functional theory provides an expression of the ground state energy of a system of interacting electrons in an external potential as a functional of the ground state electronic density [1]. Let us assume for simplicity that the spin polarization of the system of interest is identically zero. In the Kohn-Sham formulation of density functional theory 2, the ground state density is written in terms of single-particle orbitals obeying the equations in atomic units $(\hbar=e=m=1)$ :

$$
\left\{-\frac{1}{2} \nabla^{2}+v_{\mathrm{ext}}(\mathbf{r})+\int \frac{\rho\left(\mathbf{r}^{\prime}\right)}{\left|\mathbf{r}-\mathbf{r}^{\prime}\right|} \mathrm{d} \mathbf{r}^{\prime}+v_{\mathrm{xc}}([\rho] ; \mathbf{r})\right\} \psi_{i}=\epsilon_{i} \psi_{i}
$$

where

$$
\rho(\mathbf{r})=\sum_{i=1}^{N}\left|\psi_{i}(\mathbf{r})\right|^{2}
$$

The electronic density is constructed by summing over the $N$ lowest energy orbitals where $N$ is the number of electrons. $v_{\text {ext }}(\mathbf{r})$ is the external potential. The exchange-correlation potential $v_{\mathrm{xc}}([\rho] ; \mathbf{r})$ is the functional derivative of the exchange-correlation energy $E_{\mathrm{xc}}[\rho]$ that enters in the expression for the total energy of the system:

$$
E=-\frac{1}{2} \sum_{i=1}^{N} \int \psi_{i} \nabla^{2} \psi_{i} \mathrm{~d} \mathbf{r}+\int \rho(\mathbf{r}) v_{\mathrm{ext}}(\mathbf{r}) \mathrm{d} \mathbf{r}+\frac{1}{2} \iint \frac{\rho(\mathbf{r}) \rho\left(\mathbf{r}^{\prime}\right)}{\left|\mathbf{r}-\mathbf{r}^{\prime}\right|} \mathrm{d} \mathbf{r} \mathrm{d} \mathbf{r}^{\prime}+E_{\mathrm{xc}}[\rho]
$$

The exchange-correlation functional is written as the sum of two separate contributions for exchange and correlation,

$$
E_{\mathrm{xc}}[\rho]=E_{\mathrm{x}}[\rho]+E_{\mathrm{c}}[\rho] .
$$

The definition of the exchange energy is in terms of the non-interacting wave function $\Phi_{0}$, the Slater determinant constructed from the Kohn-Sham orbitals, as

$$
\mathrm{E}_{\mathrm{x}}[\rho]=\left\langle\Phi_{0}\left|V_{\mathrm{ee}}\right| \Phi_{0}\right\rangle-\frac{1}{2} \iint \frac{\rho(\mathbf{r}) \rho\left(\mathbf{r}^{\prime}\right)}{\left|\mathbf{r}-\mathbf{r}^{\prime}\right|} \mathrm{d} \mathbf{r} \mathrm{d} \mathbf{r}^{\prime},
$$

where $V_{\text {ee }}$ is the electron-electron interaction. This definition differs from the conventional quantum chemistry definition of $E_{\mathrm{x}}$ as the exchange energy in a Hartree-Fock calculation, given by the same expression as in Eq. 5 but with the Kohn-Sham determinant replaced by the Hartree-Fock determinant. The separation of the exchange-correlation functional into exchange and correlation yields a corresponding splitting of the exchange-correlation potential into $v_{\mathrm{x}}([\rho] ; \mathbf{r})$ and $v_{\mathrm{c}}([\rho] ; \mathbf{r})$. In this formulation, the essential unknown quantity is the exchange-correlation energy $E_{\mathrm{xc}}[\rho]$. If the functional form of $E_{\mathrm{xc}}[\rho]$, and consequently the exchange-correlation potential, were available, we could solve the $N$-electron problem by finding the solution of a set of single-particle equations.

The exchange functional, as defined in Eq. 5, scales under uniform density scaling, $\rho_{\lambda}(\mathbf{r})=\lambda^{3} \rho(\lambda \mathbf{r})$, 20] as 


$$
E_{\mathrm{x}}\left[\rho_{\lambda}\right]=\lambda E_{\mathrm{x}}[\rho]
$$

and its spin polarized version is simply given in terms of the unpolarized exchange functional [21] as

$$
E_{\mathrm{x}}\left[\rho_{\uparrow}, \rho_{\downarrow}\right]=\frac{1}{2}\left\{E_{\mathrm{x}}\left[2 \rho_{\uparrow}\right]+E_{\mathrm{x}}\left[2 \rho_{\downarrow}\right]\right\} .
$$

Clearly, the separation of the exchange-correlation functional according to Eq. . is useful since, for exchange, only an approximation for the unpolarized functional needs to be sought and the behavior under uniform scaling determines how derivatives of the density combine with the density in an approximate exchange functional:

$$
E_{\mathrm{x}}^{\text {approx }}[\rho]=\int \rho(\mathbf{r})^{4 / 3} F\left(|\nabla \rho(\mathbf{r})| / \rho(\mathbf{r})^{4 / 3}, \nabla^{2} \rho(\mathbf{r}) / \rho(\mathbf{r})^{5 / 3}, \ldots\right) \mathrm{d} \mathbf{r} .
$$

\section{SEPARATION OF $V_{\mathrm{XC}}$ INTO $V_{\mathrm{X}}$ PLUS $V_{\mathrm{C}}$}

For the special case of two electrons in a singlet state, the separation of the exchange-correlation potential into exchange plus correlation is quite simple since the exchange potential is simply given by the condition that it cancels the self-interaction term in the Hartree potential. On the other hand, for many-electron systems, this decomposition into exchange and correlation components has never been done. In previous work [5, 6, 9, 17, 12], the exchange potential was defined as the difference of the effective Kohn-Sham potential yielding the Hartree-Fock density and the sum of the Hartree and the external potentials. The correlation potential was then obtained as the difference of the exchangecorrelation potential corresponding to the exact density and the above approximate exchange potential. Note that this "exchange" potential is not an exchange-correlation potential since we are subtracting the wrong external potential: the Hartree-Fock density is the true ground state density for a Hamiltonian with an external potential different than the original one. However, it is also not the exchange potential corresponding to the Hartree-Fock density (although very close to it) since it is not the functional derivative with respect to the density of the exchange energy evaluated for the orbitals obtained from the effective potential yielding the Hartree-Fock density. Therefore, this separation scheme is incorrect: it involves two densities, the exact and the Hartre-Fock densities, and, moreover, the potential used for exchange is only approximately equal to the exchange potential corresponding to the Hartree-Fock density.

We follow Görling and Levy [22] in showing how to separate the exchange-correlation potential into exchange and correlation. We consider a spin unpolarized system. If we assume that the density $\rho$ is non-interacting $v$-representable, it can be expressed as in Eq. 2 in terms of single-particle orbitals $\left\{\psi_{i}\right\}$ of the Kohn-Sham potential $v_{\mathrm{s}}(\mathbf{r})$,

$$
v_{\mathrm{s}}(\mathbf{r})=v_{\mathrm{ext}}(\mathbf{r})+\int \frac{\rho\left(\mathbf{r}^{\prime}\right)}{\left|\mathbf{r}-\mathbf{r}^{\prime}\right|} \mathrm{d} \mathbf{r}^{\prime}+v_{\mathrm{xc}}([\rho] ; \mathbf{r}) .
$$

The exchange energy is a functional of the density but can also be expressed in terms of the Kohn-Sham orbitals $\left\{\psi_{i}\right\}$ (Eq. 5) as

$$
\mathrm{E}_{\mathrm{x}}[\rho]=-\frac{1}{2} \sum_{i=1}^{N} \sum_{j=1}^{N} \delta_{m_{s_{i}}, m_{s_{j}}} \iint \frac{\psi_{i}^{*}(\mathbf{r}) \psi_{j}^{*}\left(\mathbf{r}^{\prime}\right) \psi_{j}(\mathbf{r}) \psi_{i}\left(\mathbf{r}^{\prime}\right)}{\left|\mathbf{r}-\mathbf{r}^{\prime}\right|} \mathrm{d} \mathbf{r} \mathrm{d} \mathbf{r}^{\prime}
$$

where the $\delta$-function is over the spin quantum numbers of the $i$-th and $j$-th orbitals. We evaluate the functional derivative of the exchange energy functional with respect to the Kohn-Sham potential as

$$
\frac{\delta \mathrm{E}_{\mathbf{x}}[\rho]}{\delta v_{\mathrm{s}}(\mathbf{r})}=\int \frac{\delta \mathrm{E}_{\mathbf{x}}[\rho]}{\delta \rho\left(\mathbf{r}^{\prime}\right)} \frac{\delta \rho\left(\mathbf{r}^{\prime}\right)}{\delta v_{\mathrm{s}}(\mathbf{r})} \mathrm{d} \mathbf{r}^{\prime}=\int v_{\mathrm{x}}\left([\rho] ; \mathbf{r}^{\prime}\right) \sum_{i=1}^{N}\left(\psi_{i}^{*}\left(\mathbf{r}^{\prime}\right) \frac{\delta \psi_{i}\left(\mathbf{r}^{\prime}\right)}{\delta v_{\mathrm{s}}(\mathbf{r})}+\frac{\delta \psi_{i}^{*}\left(\mathbf{r}^{\prime}\right)}{\delta v_{\mathrm{s}}(\mathbf{r})} \psi_{i}\left(\mathbf{r}^{\prime}\right)\right) \mathrm{d} \mathbf{r}^{\prime} .
$$

On the other hand, since the exchange functional can be written as a function of the orbitals (Eq. 10), we also have

$$
\frac{\delta \mathrm{E}_{\mathrm{x}}[\rho]}{\delta v_{\mathrm{s}}(\mathbf{r})}=\sum_{i=1}^{N} \int\left(\frac{\delta \mathrm{E}_{\mathrm{x}}[\rho]}{\delta \psi_{i}\left(\mathbf{r}^{\prime}\right)} \frac{\delta \psi_{i}\left(\mathbf{r}^{\prime}\right)}{\delta v_{\mathrm{s}}(\mathbf{r})}+\frac{\delta \mathrm{E}_{\mathrm{x}}[\rho]}{\delta \psi_{i}^{*}\left(\mathbf{r}^{\prime}\right)} \frac{\delta \psi_{i}^{*}\left(\mathbf{r}^{\prime}\right)}{\delta v_{\mathrm{s}}(\mathbf{r})}\right) \mathrm{d} \mathbf{r}^{\prime}
$$


If we combine Eqs. 11 and 12, we obtain the integral equation

$$
\int v_{\mathrm{x}}\left([\rho] ; \mathbf{r}^{\prime}\right) \mathcal{K}\left(\mathbf{r}^{\prime}, \mathbf{r}\right) \mathrm{d} \mathbf{r}^{\prime}=\mathcal{Q}(\mathbf{r}),
$$

where the kernel $\mathcal{K}\left(\mathbf{r}^{\prime}, \mathbf{r}\right)$ and the right hand side $\mathcal{Q}(\mathbf{r})$ depend on the orbital $\left\{\psi_{i}\right\}$ and their functional derivative with respect to the potential $v_{\mathrm{S}}(\mathbf{r})$. This integral equation is equivalent to the one solved in the optimized effective potential method (OEP) where the KS orbitals are replaced by the OEP orbitals [23]. The functional derivatives of the orbitals $\delta \psi_{i}(\mathbf{r}) / \delta v_{\mathrm{s}}\left(\mathbf{r}^{\prime}\right)$ can be expressed in terms of the Green's function $G_{i}\left(\mathbf{r}, \mathbf{r}^{\prime}\right)$ as

$$
\frac{\delta \psi_{i}(\mathbf{r})}{\delta v_{\mathrm{s}}\left(\mathbf{r}^{\prime}\right)}=-G_{i}\left(\mathbf{r}, \mathbf{r}^{\prime}\right) \psi_{i}\left(\mathbf{r}^{\prime}\right)
$$

where $G_{i}\left(\mathbf{r}, \mathbf{r}^{\prime}\right)$ satisfies the differential equation

$$
\left(-\frac{1}{2} \nabla^{2}+v_{\mathbf{s}}(\mathbf{r})-\epsilon_{i}\right) G_{i}\left(\mathbf{r}, \mathbf{r}^{\prime}\right)=\delta\left(\mathbf{r}-\mathbf{r}^{\prime}\right)-\psi_{i}(\mathbf{r}) \psi_{i}^{*}\left(\mathbf{r}^{\prime}\right)
$$

By knowing the exchange-correlation potential, the KS orbitals and eigenvalues, we can compute the Green's functions $\left\{G_{i}\right\}$ and, consequently, the kernel $\mathcal{K}$ and the function $\mathcal{Q}$. If we express the exchange potential as a linear combination of basis functions, Eq. 13 can be rewritten as a non-homogeneous set of linear equations for the coefficients of the expansion of the potential in the basis set.

Once the exchange potential is determined, the correlation potential is simply obtained as the difference:

$$
v_{\mathrm{c}}([\rho] ; \mathbf{r})=v_{\mathrm{xc}}([\rho] ; \mathbf{r})-v_{\mathrm{x}}([\rho] ; \mathbf{r}) .
$$

In Appendix A, the equations derived in this section are rewritten for the case of a closed shell system.

\section{COMPARISON WITH APPROXIMATE SEPARATION SCHEMES}

For the Be atom and the Ne atom, we calculate the exchange potentials as explained in the previous section. The correlation potentials are determined as the difference of the accurate exchange-correlation potentials and the exchange components (Eq. 16). We already mentioned that, in the past, an approximate "exchange" potential was instead used, given by the effective potential yielding the Hartree-Fock density minus the Hartree and the external potentials:

$$
\tilde{v}_{\mathrm{x}}\left(\left[\rho_{\mathrm{HF}}\right] ; \mathbf{r}\right)=v_{s}\left(\left[\rho_{\mathrm{HF}}\right] ; \mathbf{r}\right)-\int \mathrm{d} \mathbf{r}^{\prime} \frac{\rho_{\mathrm{HF}}\left(\mathbf{r}^{\prime}\right)}{\left|\mathbf{r}-\mathbf{r}^{\prime}\right|}-v_{\mathrm{ext}}(\mathbf{r}),
$$

where we introduced an explicit dependence of the Kohn-Sham potential $v_{s}$ on the density reproduced by $v_{s}$. The potential $\tilde{v}_{\mathrm{x}}$ is very close to the exchange potential corresponding to the Hartree-Fock density, $v_{\mathrm{x}}\left(\left[\rho_{\mathrm{HF}}\right] ; \mathbf{r}\right)$. To determine $v_{\mathrm{x}}\left(\left[\rho_{\mathrm{HF}}\right] ; \mathbf{r}\right)$, we can use the same scheme explained in the previous section with the orbitals given by the Kohn-Sham orbitals corresponding to the effective potential yielding the Hartree-Fock density instead of the exact density. We denote by $v_{\mathrm{c}}^{\mathrm{A}}$ and $v_{\mathrm{c}}^{\mathrm{B}}$ the correlation potentials determined as the difference of the accurate $v_{\mathrm{xc}}$ and $\tilde{v}_{\mathrm{x}}\left(\left[\rho_{\mathrm{HF}}\right] ; \mathbf{r}\right)$ and $v_{\mathrm{x}}\left(\left[\rho_{\mathrm{HF}}\right] ; \mathbf{r}\right)$ respectively:

$$
v_{\mathrm{c}}^{\mathrm{A}}(\mathbf{r})=v_{\mathrm{xc}}([\rho] ; \mathbf{r})-\tilde{v}_{\mathrm{x}}\left(\left[\rho_{\mathrm{HF}}\right] ; \mathbf{r}\right), \quad v_{\mathrm{c}}^{\mathrm{B}}(\mathbf{r})=v_{\mathrm{xc}}([\rho] ; \mathbf{r})-v_{\mathrm{x}}\left(\left[\rho_{\mathrm{HF}}\right] ; \mathbf{r}\right) .
$$

As discussed in Ref. [13], for two-electron systems, $\tilde{v}_{\mathrm{x}}\left(\left[\rho_{\mathrm{HF}}\right] ; \mathbf{r}\right)=v_{\mathrm{x}}\left(\left[\rho_{\mathrm{HF}}\right] ; \mathbf{r}\right)$ and, consequently, $v_{\mathrm{c}}^{\mathrm{A}}(\mathbf{r})=v_{\mathrm{c}}^{\mathrm{B}}(\mathbf{r})$. Further, it was empirically found that the difference between $v_{\mathrm{c}}(\mathbf{r})$ and $v_{\mathrm{c}}^{\mathrm{A}, \mathrm{B}}(\mathbf{r})$ is small on the scale of $v_{\mathrm{c}}(\mathbf{r})$.

Here, we find that even for the many-electron atoms Be and Ne, the difference between $v_{\mathrm{x}}([\rho] ; \mathbf{r}), v_{\mathrm{x}}\left(\left[\rho_{\mathrm{HF}}\right] ; \mathbf{r}\right)$ and $\tilde{v}_{\mathrm{x}}\left(\left[\rho_{\mathrm{HF}}\right] ; \mathbf{r}\right)$ are almost not visible on the scale of $v_{\mathrm{x}}([\rho] ; \mathbf{r})$. As shown in Fig. 1, the difference between $v_{\mathrm{c}}^{\mathrm{A}}(\mathbf{r})$ and $v_{\mathrm{c}}^{\mathrm{B}}(\mathbf{r})$ is just barely visible even on the more expanded scale of $v_{\mathrm{c}}(\mathbf{r})$, This agreement is expected since the HF and the OEP densities are very close to each other and, for the OEP density, the agreement would be perfect. On the 
other hand, the difference between either $v_{\mathrm{c}}(\mathbf{r})$ and $v_{\mathrm{c}}^{\mathrm{A}}(\mathbf{r})$ or $v_{\mathrm{c}}(\mathbf{r})$ and $v_{\mathrm{c}}^{\mathrm{B}}(\mathbf{r})$ is visible. For both atoms, the exact and approximate potentials are clearly different, although the shapes are very similar. The similarity of the exact and the approximate potentials justifies the use of the approximate scheme used in earlier work.
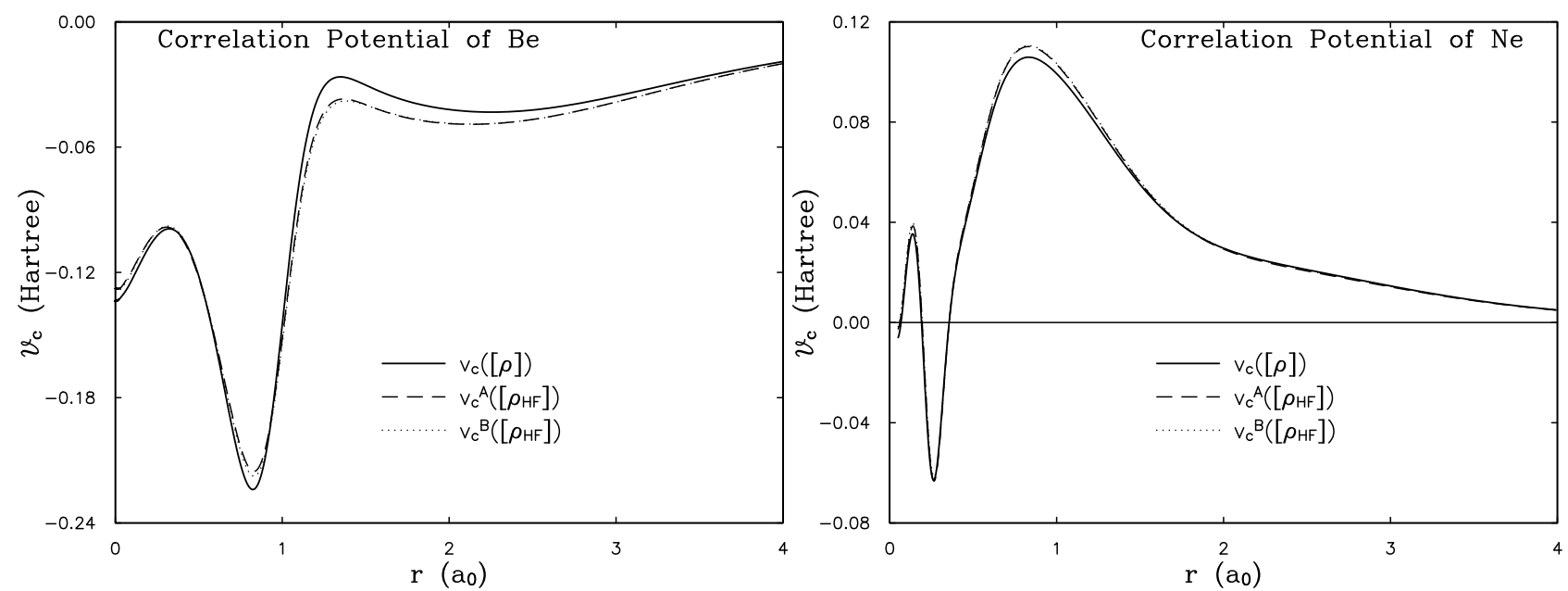

FIG. 1. Comparison of the correlation potentials of Be and Ne. $v_{\mathrm{c}}$ is the correct correlation potential from Eq. 16, $v_{\mathrm{c}}^{\mathrm{A}}(\mathbf{r})$ and $v_{\mathrm{c}}^{\mathrm{B}}(\mathbf{r})$ are the approximate correlation potentials constructed from the HF density using Eq. 18. $v_{\mathrm{c}}^{\mathrm{A}}(\mathbf{r})$ and $v_{\mathrm{c}}^{\mathrm{B}}(\mathbf{r})$ are nearly indistinguishable on the scale of these plots. For Ne, there is some uncertainty in the potentials for $r<0.4 a_{0}$.

\section{ACKNOWLEDGEMENTS}

We benefited from useful discussions with Mel Levy, Andreas Görling and Eberhard Gross. The calculations were performed on the IBM SP2 computer at the Cornell Theory Center. This work is supported by the Office of Naval Research and NATO (grant number CRG 940594). X.G. acknowledges financial support from FNRS-Belgium and the European Union (Human Capital and Mobility Program contract CHRX-CT940462).

\section{APPENDIX A: CLOSED-SHELL CASE}

In Sec. II. we presented the theory of separation of the exchange-correlation potential into the exchange and correlation components for spin-unpolarized systems. In the present section, we restrict ourselves to the special case of a closed shell atom. For a closed shell atom, the self-consistent solutions of the Kohn-Sham equations (Eqs. 1 and 2) can be factorized as the product of radial and angular components:

$$
\psi_{i}(\mathbf{r})=R_{i}(r) Y_{l_{i} m_{i}}(\hat{\mathbf{r}})=\frac{\phi_{i}(r)}{r} Y_{l_{i} m_{i}}(\hat{\mathbf{r}}),
$$

where $l_{i}$ is the angular momentum quantum number. Using this expression for the orbitals, the density (Eq. 2) can be rewritten as a sum over the occupied shells:

$$
\rho(r)=\frac{1}{4 \pi r^{2}} \sum_{i=1}^{N_{s}} f_{i} \phi_{i}^{2}(r)
$$

where $N_{s}$ is the number of occupied shells and $f_{i}$ is the occupation number of the $i$-th shell, $f_{i}=2\left(2 l_{i}+1\right)$.

Following the derivation by Slater [24], we rewrite the exchange energy (Eq. 10) as

$$
E_{\mathrm{x}}[\rho]=-\sum_{i, j}^{N_{s}} \sqrt{\left(2 l_{i}+1\right)\left(2 l_{j}+1\right)} \sum_{k=\left|l_{i}-l_{j}\right|}^{l_{i}+l_{j}} c^{k}\left(l_{i}, 0 ; l_{j}, 0\right) G^{k}\left(n_{i}, l_{i} ; n_{j}, l_{j}\right),
$$


where the coefficients $c^{k}$ incorporate the integrals over $\theta$ and are tabulated in Ref. [24] and $G^{k}$ is given by

$$
G^{k}\left(n_{i}, l_{i} ; n_{j}, l_{j}\right)=\int \mathrm{d} r_{1} \int \mathrm{d} r_{2} \phi_{i}\left(r_{1}\right) \phi_{j}\left(r_{2}\right) \phi_{j}\left(r_{1}\right) \phi_{i}\left(r_{2}\right) \frac{r_{<}^{k}}{r_{>}^{k+1}},
$$

with $r_{<}=\min \left\{r_{1}, r_{2}\right\}$ and $r_{>}=\max \left\{r_{1}, r_{2}\right\}$.

The functional derivative of the exchange energy with respect to the effective Kohn-Sham potential (Eqs. 11 and 12) can here be obtained taking into account that the density depends only on the radial components of the Kohn-Sham orbitals. Eq. 11 is therefore equivalent to

$$
\frac{\delta E_{\mathrm{x}}[\rho]}{\delta v_{s}(r)}=2 \int_{0}^{\infty} \mathrm{d} r^{\prime} v_{\mathrm{x}}\left(r^{\prime}\right) \sum_{i=1}^{N_{s}} f_{i} \phi_{i}\left(r^{\prime}\right) \frac{\delta \phi_{i}\left(r^{\prime}\right)}{\delta v_{s}(r)}
$$

while Eq. 12 reduces to

$$
\frac{\delta E_{\mathrm{x}}[\rho]}{\delta v_{s}(r)}=\int_{0}^{\infty} \mathrm{d} r^{\prime} \sum_{i=1}^{N_{s}} \frac{\delta E_{\mathrm{x}}[\rho]}{\delta \phi_{i}\left(r^{\prime}\right)} \frac{\delta \phi_{i}\left(r^{\prime}\right)}{\delta v_{s}(r)}
$$

Eqs. A5 and A6 can be combined to give the following integral equation for the exchange potential:

$$
\int_{0}^{\infty} \mathrm{d} r^{\prime} v_{\mathrm{x}}\left(r^{\prime}\right) \mathcal{K}\left(r^{\prime}, r\right)=\mathcal{Q}(r)
$$

The functional derivative of the exchange energy (Eq. A3) with respect to the radial orbital is given by

$$
\frac{\delta E_{\mathbf{x}}[\rho]}{\delta \phi_{i}(r)}=-2 f_{i} \sum_{j=1}^{N_{s}} \sqrt{\frac{2 l_{j}+1}{2 l_{i}+1}} \sum_{k=\left|l_{i}-l_{j}\right|}^{l_{i}+l_{j}} c^{k}\left(l_{i}, 0 ; l_{j}, 0\right) \phi_{j}(r) \int_{0}^{\infty} d r_{2} \phi_{i}\left(r_{2}\right) \phi_{j}\left(r_{2}\right) \frac{r_{<}^{k}}{r_{>}^{k+1}} .
$$

The functional derivative of the radial orbital, $\delta \phi_{i}(r) / \delta v_{s}\left(r^{\prime}\right)$, is expressed in terms of the Green's function $G_{i}\left(r, r^{\prime}\right)$ as

$$
\frac{\delta \phi_{i}(r)}{\delta v_{s}\left(r^{\prime}\right)}=-G_{i}\left(r, r^{\prime}\right) \phi_{i}\left(r^{\prime}\right)
$$

where $G_{i}\left(r, r^{\prime}\right)$ satisfies the following differential equation:

$$
\left\{-\frac{1}{2} \frac{\mathrm{d}^{2}}{\mathrm{~d} r^{2}}+\frac{l_{i}\left(l_{i}+1\right)}{2 r^{2}}+v_{s}(r)-\epsilon_{i}\right\} G_{i}\left(r, r^{\prime}\right)=\delta\left(r-r^{\prime}\right)-\phi_{i}(r) \phi_{i}\left(r^{\prime}\right) .
$$

This equation can also be derived by starting from Eq. 15 in polar coordinates and projecting out the radial component. It can be easily checked that $G_{i}$ has the following expression:

$$
G_{i}\left(r, r^{\prime}\right)=\sum_{j \neq i: l_{j}=l_{i}} \frac{\phi_{j}(r) \phi_{j}\left(r^{\prime}\right)}{\epsilon_{j}-\epsilon_{i}}
$$

where the sum is over all the orbitals, except the $i$-th one, with angular momentum quantum number $l_{i}$. In solving the differential equation for $G_{i}$, we set $r \neq r^{\prime}$, divide by $\phi_{i}\left(r^{\prime}\right)$ and determine $\chi_{\text {out }}(r)$ and $\chi_{\text {in }}(r)$ as solutions of outward $\left(r<r^{\prime}\right)$ and inward $\left(r>r^{\prime}\right)$ integration. $\phi(r)$ is a homogenous solution of Eq. A10 and can be added to $\chi_{\text {out }}(r)$ and $\chi_{\text {in }}(r)$ as $\alpha_{\text {out }} \phi(r)$ and $\alpha_{\text {in }} \phi(r)$ respectively. The difference $\alpha_{\text {out }}-\alpha_{\text {in }}$ is determined by imposing continuity on $G_{i}$ and the sum $\alpha_{\text {out }}+\alpha_{\text {in }}$ by requiring that

$$
\int_{0}^{\infty} \mathrm{d} r \phi_{i}(r) \frac{\delta \phi_{i}(r)}{\delta v_{s}\left(r^{\prime}\right)}=0
$$


which follows from the normalization of $\phi_{i}(r)$. Finally, we obtain

$$
\frac{\delta \phi_{i}(r)}{\delta v_{s}\left(r^{\prime}\right)}=\tilde{G}_{i}\left(r, r^{\prime}\right)-\phi_{i}(r) \int_{0}^{\infty} \mathrm{d} r^{\prime \prime} \phi_{i}\left(r^{\prime \prime}\right) \tilde{G}_{i}\left(r^{\prime \prime}, r^{\prime}\right),
$$

where $\tilde{G}_{i}\left(r, r^{\prime}\right)$ is

$$
\begin{aligned}
\tilde{G}_{i}\left(r, r^{\prime}\right) & =\theta\left(r-r^{\prime}\right)\left\{\chi_{\mathrm{in}}(r) \phi_{i}\left(r^{\prime}\right)^{2}-\frac{1}{2}\left[\chi_{\mathrm{in}}\left(r^{\prime}\right)-\chi_{\mathrm{out}}\left(r^{\prime}\right)\right] \phi_{i}(r) \phi_{i}\left(r^{\prime}\right)\right\} \\
& +\theta\left(r^{\prime}-r\right)\left\{\chi_{\mathrm{out}}(r) \phi_{i}\left(r^{\prime}\right)^{2}+\frac{1}{2}\left[\chi_{\mathrm{in}}\left(r^{\prime}\right)-\chi_{\mathrm{out}}\left(r^{\prime}\right)\right] \phi_{i}(r) \phi_{i}\left(r^{\prime}\right)\right\} .
\end{aligned}
$$

[1] P. Hohenberg and W. Kohn, Phys. Rev. 136, B864 (1964).

[2] W. Kohn and L. J. Sham, Phys. Rev. 140, A1133 (1976).

[3] D. W. Smith, S. Jagannathan, and G. S. Handler, Int. J. Quantum Chem. Symp. 13, 103 (1979).

[4] U. von Barth in Electronic Structure of Complex Systems, edited by P. Phariseau and W. M. Temmerman, NATO, ASI Series B, vol. 113 (1984).

[5] C. -O. Almbladh and A. C. Pedroza, Phys. Rev. B 29, 2322 (1984); A. C. Pedroza, Phys. Rev. A 33, 804 (1986).

[6] F. Aryasetiawan and M. J. Stott, Phys. Rev. B 34, 4401 (1986); ibid. 38, 2974 (1988).

[7] A. Nagy and N. H. March, Phys. Rev. A 39, 5512 (1989); ibid. 40, 554 (1989).

[8] E. R. Davidson, Int. J. Quant. Chem. 37, 811 (1990).

[9] J. Chen, R. O. Esquivel, and M. J. Stott, Philos. Mag. B 69, 1001 (1994).

[10] R. van Leeuwen and E. J. Baerends, Phys. Rev. A 49, 2421 (1994).

[11] R. C. Morrison and Q. Zhao, Phys. Rev. A 51, 1980 (1995).

[12] E. V. Ludeña, J. Maldonado, R. López-Boada, T. Koga, and E. S. Kryachko, J. Chem. Phys. 102, 318 (1995).

[13] C. J. Umrigar and X. Gonze, Phys. Rev. A 50, 3827 (1994).

[14] M. A. Buijse, E. J. Baerends and J. G. Snijders, Phys. Rev. A 40, 4190 (1989).

[15] O. V. Gritsenko, R. van Leeuwen and E. J. Baerends, Phys. Rev. A 52, 1870 (1995).

[16] V. E. Ingamells and N. C. Handy, Chem. Phys. Lett. 248, 373 (1996).

[17] C. J. Umrigar and X. Gonze, in High Performance Computing and its Application to the Physical Sciences, proceedings of the Mardi Gras '93 Conference, edited by D. A. Browne et al., (World Scientific, Singapore, 1993).

[18] W. Knorr and R. W. Godby, Phys. Rev. Lett. 68, 639 (1992).

[19] C. Umrigar and X. Gonze, unpublished (1996).

[20] M. Levy and J. P. Perdew, Phys. Rev. A 32, 2010 (1985).

[21] G. L. Oliver and J. P. Perdew, Phys. Rev. A 20, 397 (1979).

[22] A. Görling and M. Levy, Int. J. Quant. Chem, Symp. 29, 93 (1995); Phys. Rev. A 50, 196 (1994).

[23] R. T. Sharp and G. K. Horton, Phys. Rev. 90, 317 (1953); J. D. Talman, and W. F. Shadwick, Phys. Rev. A 14, 36 (1976).

[24] J. C. Slater, Quantum Theory of Atomic structure, Vol. I (McGraw-Hill, New York, 1960) 